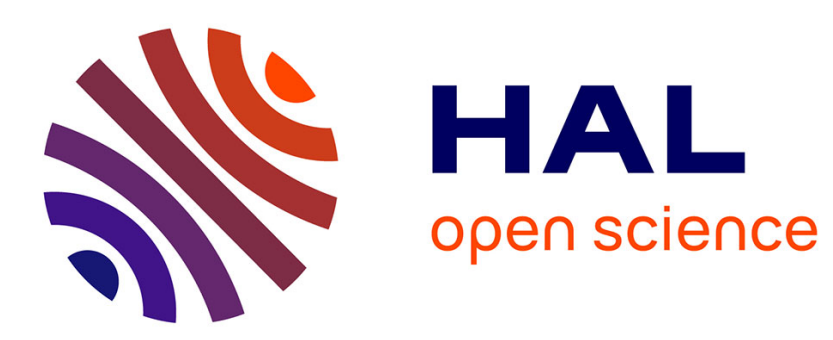

\title{
Le concept d'Aurignacien : entre rupture préhistorique et obstacle épistémologique
}

Nicolas Teyssandier, Despina Liolios

\section{To cite this version:}

Nicolas Teyssandier, Despina Liolios. Le concept d'Aurignacien: entre rupture préhistorique et obstacle épistémologique. Bulletin de la Société préhistorique française, 2008, 105 (4), pp.737-747. 10.3406/bspf.2008.13782 . halshs-00430890

\section{HAL Id: halshs-00430890 \\ https://shs.hal.science/halshs-00430890}

Submitted on 21 Oct 2021

HAL is a multi-disciplinary open access archive for the deposit and dissemination of scientific research documents, whether they are published or not. The documents may come from teaching and research institutions in France or abroad, or from public or private research centers.
L'archive ouverte pluridisciplinaire HAL, est destinée au dépôt et à la diffusion de documents scientifiques de niveau recherche, publiés ou non, émanant des établissements d'enseignement et de recherche français ou étrangers, des laboratoires publics ou privés. 


\title{
Le concept d'Aurignacien : entre rupture préhistorique et
} obstacle épistémologique

\author{
Nicolas Teyssandier, Despina Liolios
}

\begin{abstract}
The Aurignacian is a historical concept, defined in a masterly manner by $\mathrm{H}$. Breuil in the early twentieth century. Using $\mathrm{H}$. Breuil's original conceptions as a starting point, we shall attempt to show that our notions about what supposedly characterizes this technocomplex are constraining and actually operate as epistemological obstacles. With the help of some examples drawn from current research, we shall discuss the discrepancy between the archaeological data and the major component of the Aurignacian interpretive frame, namely, that the Aurignacian is thought to be a homogeneous and exogenous culture, pregnant with the incipient defining traits of the Upper Palaeolithic.
\end{abstract}

\section{Résumé}

L'Aurignacien est un concept historique magistralement mis en place par $\mathrm{H}$. Breuil au début du XXe siècle. En repartant de la conception que $\mathrm{H}$. Breuil a défendue, nous essaierons de montrer comment nos représentations de ce qui caractérise ce technocomplexe fonctionnent aujourd'hui comme des obstacles épistémologiques. À travers quelques exemples pris dans les recherches récentes, nous discuterons de la discordance entre les données archéologiques et les principaux éléments du paradigme interprétatif dominant de l'Aurignacien, le considérant comme une culture homogène et exogène, au travers de laquelle sont inaugurés les caractères constitutifs du Paléolithique supérieur.

\section{Citer ce document / Cite this document :}

Teyssandier Nicolas, Liolios Despina. Le concept d’Aurignacien : entre rupture préhistorique et obstacle épistémologique. In: Bulletin de la Société préhistorique française, tome 105, $n^{\circ} 4,2008$. pp. 737-747;

doi : https://doi.org/10.3406/bspf.2008.13782

https://www.persee.fr/doc/bspf_0249-7638_2008_num_105_4_13782

Fichier pdf généré le 08/09/2020 
Nicolas TEYSSANDIER et Despina LIOLIOS

\title{
Le concept d'Aurignacien : entre rupture préhistorique et obstacle épistémologique
}

\begin{abstract}
Résumé
L'Aurignacien est un concept historique magistralement mis en place par $H$. Breuil au début du XX $X^{e}$ siècle. En repartant de la conception que $H$. Breuil a défendue, nous essaierons de montrer comment nos représentations de ce qui caractérise ce technocomplexe fonctionnent aujourd'hui comme des obstacles épistémologiques. À travers quelques exemples pris dans les recherches récentes, nous discuterons de la discordance entre les données archéologiques et les principaux éléments du paradigme interprétatif dominant de l'Aurignacien, le considérant comme une culture homogène et exogène, au travers de laquelle sont inaugurés les caractères constitutifs du Paléolithique supérieur.
\end{abstract}

\section{Abstract}

The Aurignacian is a historical concept, defined in a masterly manner by $H$. Breuil in the early twentieth century. Using H. Breuil's original conceptions as a starting point, we shall attempt to show that our notions about what supposedly characterizes this technocomplex are constraining and actually operate as epistemological obstacles. With the help of some examples drawn from current research, we shall discuss the discrepancy between the archaeological data and the major component of the Aurignacian interpretive frame, namely, that the Aurignacian is thought to be a homogeneous and exogenous culture, pregnant with the incipient defining traits of the Upper Palaeolithic.

UN PARADIGME TRÈS PUISSANT : LA PENSÉE DE H. BREUIL, APPORTS ET OBSTACLES

Depuis les premiers moments de sa conception, l'Aurignacien occupe un rôle déterminant dans l'histoire du Paléolithique. Cet article tente d'interroger cette notion sous l'angle des problèmes interprétatifs liés à sa construction et à son utilisation. Il ne s'agit en aucun cas d'une chronique historiographique, mais d'un moment de prise de recul par rapport à notre objet d'étude.

Le paradigme qui a prévalu jusqu'à une période récente voyait dans l'Aurignacien la première culture européenne propre à l'Homme moderne; ce paradigme a été en grande partie édifié selon le cadre proposé par
H. Breuil (1913) au début du $\mathrm{XX}^{\mathrm{e}}$ siècle. Et l'on comprend aisément pourquoi l'Aurignacien a su cristalliser, dès cette époque, une grande attention. En effet, comment ne pas voir dans les innovations techniques qui le caractérisent, pièces d'art mobilier et autres parures, la preuve manifeste de ce que par ailleurs l'évolutionnisme revendiquait depuis longtemps : l'évolution de la vie va du simple au complexe, de l'homogène à l'hétérogène, du continu au discontinu. $\mathrm{H}$. Breuil adhérait pleinement à cette représentation progressiste, tant il voyait dans l'évolutionnisme le résultat de la démarche scientifique par excellence. Dans sa leçon d'ouverture à la chaire de Préhistoire du Collège de France, en décembre 1929, n'avait-il pas écrit : "Toute réalité dérive au moins en bonne partie de ses antécédents et devient également en partie le principe des réalités qui suivent. C'est là non une 
hypothèse mais une méthode extraordinairement féconde de connaissance. Sans ce principe radical qui n'est autre que l'idée évolutionniste, aucune science véritable du passé quel qu'il soit n'existe» (Breuil, 1929, p. 103)? Cette citation de H. Breuil en dit long sur le contexte intellectuel servant à la construction de son cadre des temps paléolithiques. Et l'on sait également que ce fut un enjeu vital pour la préhistoire naissante que d'inscrire l'histoire de l'Homme dans une histoire naturelle, de manière à l'étudier avec les outils propres aux sciences de la nature et à le dégager de sa gangue métaphysique et théologique. En définitive, quoi de plus légitime que cette posture épistémologique qui tentait de fonder la Préhistoire comme discipline véritablement scientifique?

$\mathrm{Si} \mathrm{H}$. Breuil situe ses propres travaux dans la «doctrine évolutionniste», il va toutefois rompre avec l'idée de progrès telle qu'elle était conçue au XIX ${ }^{\mathrm{e}}$ siècle et utilisée en préhistoire par G. de Mortillet notamment (Mortillet, 1872 et 1885). Le concept qui organisait jusqu'alors les représentations préhistoriennes était celui de progrès universel et continu; il supposait le perfectionnement des industries au fil du temps (Coye, 1997; Guillomet-Malmassari, 2006). Or, au tout début $\mathrm{du} \mathrm{XX}^{\mathrm{e}}$ siècle, après la disparition de G. de Mortillet et l'essor de la méthode stratigraphique, cette vision va être remise en cause (Coye, ibid.). La reconnaissance d'un Aurignacien pré-solutréen (Breuil, 1907) participe activement à ce chavirement et contribue aux refontes méthodologiques de la discipline qui se nouent à l'aube du siècle dernier. La célèbre «bataille aurignacienne» qui opposa H. Breuil aux défenseurs des positions de G. de Mortillet demeure à ce titre fondatrice de la préhistoire moderne telle qu'on l'entend pour la première moitié du $\mathrm{XX}^{\mathrm{e}}$ siècle (Dubois et Bon, 2006). En montrant que l'observation stratigraphique devait sur ce point prévaloir sur les présupposés théoriques, H. Breuil, tout en acceptant l'idée générale de progrès, va néanmoins démontrer que des moments d'arrêt ou de régression ont pu scander cette évolution et, par conséquent, ouvrir un champ de réflexion sur la rupture et la discontinuité dans l'histoire culturelle et technique (Guillomet-Malmassari, ibid.). C'est là très certainement l'un des points les plus remarquables du cadre élaboré par H. Breuil sur les débuts du Paléolithique supérieur européen : l'apparition de la notion de rupture dans l'évolution technique et culturelle paléolithique. Elle s'accompagnera, quelques années plus tard, de l'édification de la notion de «culture préhistorique » au détriment de celle d' «âge» ou d' «époque», ainsi que de scénarios évolutifs buissonnants (p. e. coexistence possible de civilisations différentes; Breuil, 1913). Peu à peu, le discours naturaliste sera délaissé au profit d'une approche historique et événementielle, composée d'acteurs et de civilisations se déplaçant et interagissant les uns vis-à-vis des autres.

L'apport théorique de la pensée de H. Breuil sera exceptionnel, mais les caractères qui lui permettront de fonder l'Aurignacien et de proposer une nouvelle classification des temps paléolithiques vont par la suite fonctionner comme autant d'obstacles épistémologiques (Bachelard, 1934). Ces caractères sont, notamment, l'idée d'une rupture essentielle entre le Paléolithique moyen et le Paléolithique supérieur, analogue à celle qui fondera l'avènement du Néolithique et l'association de ce changement culturel à l'essor d'un type humain exogène : «Il ne semble pas qu' on puisse admettre que le Paléolithique supérieur soit nulle part, dans les régions indiquées (Europe atlantique et méditerranéenne), dérivé du Moustérien. Il s'agit plus vraisemblablement d'invasions de peuples beaucoup plus élevés dans l'échelle des races et dans celle de la civilisation que leurs prédécesseurs néanderthaliens. [...] Dans l'état actuel de nos connaissances, il paraît établi que l'arrivée des Paléolithiques supérieurs ait amené, à la fin du Moustérien, un changement social et industriel et une substitution de race humaine si profonde, qu'il serait légitime, dans une classification bien coordonnée, de séparer le Paléolithique ancien des temps qui le suivent par une coupure de grandeur égale à celle qui sépare ceux-ci de l'époque néolithique ${ }^{1}$ (Breuil, 1913, p. 174). Si on y associe le paradigme évolutionniste, apparaît également une autre idée forte : l'Aurignacien porte en lui le germe de tout ce qui caractérisera le Paléolithique supérieur. H. Breuil fait naturellement référence aux manifestations artistiques, au développement et à la généralisation de la parure et des industries en matières dures animales ou encore aux transformations des industries lithiques qui, pour reprendre ses termes, se leptolithisent et s'orientent désormais vers des productions élancées de lames et de lamelles. L'ampleur des travaux de H. Breuil et l'éclairage qu'ils apportaient aux données françaises ont conduit bien d'autres spécialistes à utiliser sa classification du Paléolithique supérieur dans des régions fort éloignées de la France (voir p. e. Schmidt, 1912); si l'on peut prendre la mesure du progrès qu'il a impulsé dans d'autres régions européennes, on prend également celle d'un conditionnement certain des données.

Cette description, pour ne pas dire cette signification de l'Aurignacien, a traversé le siècle dernier et perdure encore dans l'esprit de beaucoup. Et l'Aurignacien de H. Breuil aura pu être largement amputé, perdant notamment ses phases inférieures et supérieures, dont la première finira par devenir une culture présentée comme l'une des dernières expressions des sociétés néandertaliennes, rien n'y fait : l'Aurignacien poursuit sa destinée. Notre pensée est aujourd'hui aux prises avec ses nombreuses implications. En effet, on ne retient souvent de l'Aurignacien que ce qui le lie à ce qui suivra et le différencie de ce qui le précéda. On s'empêche alors de penser ses spécificités et ses liens avec ce qui précède; on s'empêche également de penser l'hétérogénéité de l'histoire des sociétés humaines du Paléolithique supérieur. Et n'est-ce pas d'ailleurs de cette représentation dont se nourrit l'usage récent dans les débats du concept de modernité culturelle ? L'apparition de l'art, du langage, de la pensée religieuse semble s'articuler à la façon d'un grade ou d'un jalon évolutif unifiant les pratiques des Homo sapiens et gommant la diversité de signification des productions symboliques et culturelles. Ainsi, l'idée d'une origine exogène des populations aurignaciennes, distinctes des Néandertaliens et portant avec elles une «civilisation» 
nouvelle et en progrès, a fait obstacle à celle, rendue tout aussi plausible par l'avancée des recherches, d'une contribution des sociétés du Paléolithique moyen à la formation de l'Aurignacien. Au contraire, l'idée a longtemps perduré que l'Aurignacien fut une culture puissante, homogène et unitaire, au point d'apparaître dans la littérature comme la première culture paneuropéenne du Paléolithique (Kozlowski, 1993). On peut se demander aujourd'hui, à la lumière des travaux récents, si la controverse qui porte sur ses relations avec les industries dites de transition, notamment le Châtelperronien (voir par exemple Demars et Hublin, 1989; Mellars, 1989 et 2004; d'Errico et al., 1998), n'a pas eu pour effet d'accentuer ses caractères conquérants, la puissance de l'invasion aurignacienne rendant en effet plausible sa capacité à acculturer les populations autochtones, derniers représentants de sociétés néandertaliennes en voie d'extinction.

La force du modèle unitaire a par ailleurs exclu des interprétations globales des industries apparaissant trop «atypiques » ou ne correspondant pas au cadre de référence du Sud-Ouest français, qui avaient pourtant servi à $\mathrm{H}$. Breuil comme à ses successeurs, à l'instar de D. Peyrony. Cet état de fait a ainsi entravé la construction d'un Aurignacien plus polymorphe, malgré les nombreuses publications allant dans ce sens (voir p. e. Laplace, 1966; Oliva, 1989; Valoch, 1990; Straus, 1996 et 2003 ; Cabrera Valdès et al., 2001). Depuis les années quatre-vingt, les modèles dominants demeurent étroitement migrationnistes alors que les publications plus locales ou régionales décrivent fréquemment des configurations plus polymorphes, en lien avec les substrats locaux. Mais ce polymorphisme aurait sans doute été incompatible avec une autre représentation qui unit de façon univoque l'Aurignacien et l'Homme anatomiquement moderne, censé avoir migré à cette période et pas avant en Europe, censé n'avoir produit que l'Aurignacien et non d'autres industries dites de transition, censé l'avoir fait seul, sans mixité culturelle et anthropologique avec les autochtones néandertaliens. Il est intéressant de noter que cette interprétation demeure, alors même que des populations néandertaliennes sont attestées depuis environ $60000 \mathrm{BP}$ au Proche-Orient et dans le Sud de l'Asie centrale, qui figurent pourtant parmi les régions souvent proposées comme souches de l'Aurignacien (voir p. e. Kozlowski et Otte, 2000).

Cet état de fait illustre comment le paradigme dominant d'interprétation de l'Aurignacien, conçu par H. Breuil, s'est construit et précisé dans le sillon du courant évolutionniste tout au long du siècle dernier. Il a largement été entretenu, voire renforcé, par l'usage de certains outils d'analyse : d'abord, l'usage exclusif de la typologie et des comparaisons tissées à grande distance mais souvent mal maîtrisées sur un plan méthodologique. Combien de sites ont, par exemple, été attribués à l'Aurignacien ancien (I) sur la base de la présence d'une unique pointe à base fendue (Liolios, 1999 et 2006) ? Combien d'autres sont venus alimenter le débat alors même que leurs ensembles archéologiques semblaient pollués, mais comptaient quelques types aurignacoïdes, des «grattoirs » épais carénés ou à museau le plus souvent (voir par exemple Zilhão et d'Errico, 1999, 2003a et b; Teyssandier, 2003 et 2007) ? Enfin, rappelons que ce modèle de peuplement de l'Europe par l'Homme moderne et des dates anciennes autour de 40000 BP sur certains gisements ont appuyé l'idée d'un gradient est/ouest de cette diffusion sur la seule base d'une poignée de sites. Pour ne citer que les plus célèbres, Bacho Kiro en Bulgarie, Istàllòskö en Hongrie, Willendorf II en Autriche et les grottes du Jura souabe en Allemagne servent alors de points intermédiaires pour relier le berceau oriental aux centres de développement majeurs que sont le Bassin aquitain et le massif pyrénéen. Les gisements de référence n'étaient non seulement pas nombreux, mais présentaient tous des problèmes rendant leur stricte association à l'Aurignacien et à l'Homme moderne délicate (voir p. e. Zilhão et d'Errico, 1999; Teyssandier, 2007).

Ces problèmes relevaient pour certains de la datation des dépôts. Le poids accordé à des dates obtenues anciennement a souvent été démesuré; que l'on se souvienne de l'exemple d'Istàllòskö et de ses deux mesures ${ }^{14} \mathrm{C}$ d'un niveau à pointes à base fendue aux alentours de 40000 BP. Depuis, de nouvelles dates plus cohérentes avec ce contexte archéologique, autour de 33000 BP, ont été obtenues (Adams et Ringer, 2004) et une nouvelle analyse des ensembles lithiques a mis en évidence des mélanges entre l'Aurignacien et une occupation szélétienne sous-jacente (Teyssandier et al., en préparation). D'autres touchaient à la définition des ensembles archéologiques. En effet, caractériser un ensemble comme Aurignacien impliquait que son auteur ait été l'Homme moderne. De nombreux ensembles archéologiques acceptés sans discussion comme «Aurignacien très ancien » dans les années 1970-1990 ont ainsi été récemment discutés; on peut citer pour exemple Bacho Kiro couche 11 en Bulgarie (Rigaud, 2001; Tsanova et Bordes, 2003; Teyssandier, 2003, 2006 et 2007; Kozlowski, 2004; Rigaud et Lucas, 2006 ; Tsanova, 2006), Istàllòskö en Hongrie (Zilhão et d'Errico, 1999; Teyssandier, 2003 et 2007; Teyssandier et al., en préparation), Willendorf II couches 2 et 3 en Autriche (Haesaerts et Teyssandier, 2003; Teyssandier, ibid.), Vedrovice 2 en République tchèque (Zilhão et d'Errico, 1999; Teyssandier, 2007), Geissenklösterle III en Allemagne (Zilhão et d'Errico, 1999, 2003a et b; Teyssandier, 2003 et 2007; Teyssandier et Liolios, 2003; Teyssandier et al., 2006) ou le Trou Magrite en Belgique (Zilhão et d'Errico, 1999). Tous présentaient une proportion plus ou moins importante d'objets aurignacoïdes, composée notamment de grattoirs épais ou de lames retouchées. Or «aurignacoïde» ne rime pas forcément avec «Aurignacien» et encore moins avec «Homme anatomiquement moderne». Le cas de la couche 11 de Bacho Kiro est un bon exemple de basculement interprétatif suite aux réévaluations technologiques de l'assemblage (Teyssandier, 2003, 2006 et 2007; Tsanova et Bordes, 2003 ; Rigaud et Lucas, 2006; Tsanova, 2006). De porte d'entrée de l'Aurignacien en Europe, Bacho Kiro est devenu un point parmi d'autres sur une carte de l'Eurasie, à un moment où des changements techno-économiques similaires et strictement 
antérieurs au premier Aurignacien s'opèrent en diverses régions. Ancrées dans un substrat conceptuel Levallois, les productions lithiques s'allongent, des pointes sont recherchées, les outils d'extrémité se multiplient et, localement, les registres économiques peuvent évoluer, comme c'est le cas à Bacho Kiro où la couche 11 tranche avec les occupations précédentes du Paléolithique moyen récent par l'apport massif de supports et/ou outils en silex exogènes importés.

Les problèmes touchant à l'interprétation du premier Aurignacien ont déjà été abordés en détail dans plusieurs travaux récents (voir par exemple Zilhão et d'Errico, 1999, 2003a et b ; Bon, 2002; Bordes, 2002; Straus, 2003; Teyssandier, 2003, 2006 et 2007). Une relecture plus critique des données depuis une petite dizaine d'années a en effet conduit à modifier notre perception de ses premières expressions. Ainsi, la compréhension des débuts de l'Aurignacien s'est transformée sous l'effet de plusieurs travaux conduits à la fois en Europe occidentale (voir p. e. Bon, 2002; Bordes, 2002 et 2006), centrale (Conard et Bolus, 2003; Teyssandier 2003, 2006, 2007; Teyssandier et al., 2006), dans les Balkans (Teyssandier, ibid.; Tsanova, 2006) et au Proche-Orient (Goring-Morris et Belfer-Cohen dir., 2003). Nous retracerons ici schématiquement les principaux points de refonte des interprétations puis soulignerons combien les obstacles déjà évoqués demeurent néanmoins difficiles à dépasser.

\section{REFONTE DU PARADIGME SUR LA BASE DES DONNÉES RÉCENTES}

\section{Quelle unité de l'Aurignacien?}

Ces travaux discutent tout d'abord de l'unité des productions des premiers temps de l'Aurignacien, à partir des séquences de référence et sur une large zone géographique. En effet, et c'est bien là l'apport principal de ces travaux récents, l'acquisition de données factuelles avait quelque peu été mise de côté depuis le milieu des années quatre-vingt, la communauté internationale se concentrant alors en large partie sur la création de modèles globaux, basés notamment sur le produit des fouilles conduites jusqu'à la fin des années soixante-dix. En France, si de nouvelles fouilles étaient initiées (Esquicho Grapaou et la Laouza par F. Bazile, le Flageolet par J.-P. Rigaud, Brassempouy par H. Delporte p. e.), le temps n'était pas encore venu de déconstruire les paradigmes dominants et l'on cherchait plutôt à insérer ces données à l'intérieur des cadres déjà définis.

Aujourd'hui, on reconnaît l'existence d'au moins deux traditions techniques distinctes pour les débuts de l'Aurignacien: le faciès princeps, l'Aurignacien ancien (I) et des industries à grandes lamelles retouchées dites Protoaurignacien (ou Aurignacien archaïque). Cette partition n'est pas nouvelle en soi puisqu'on a depuis longtemps décrit ce Protoaurignacien sur tout le pourtour ouest-méditerranéen (Laplace, 1966). Bien que reconnu également dans le domaine atlantique (Laplace, 1966), c'est la tradition des études italiennes et espagnoles, inspirées des travaux de
G. Laplace alors que ces derniers étaient, au contraire, écartés en France, qui a conféré à son Protoaurignacien cet ancrage régional. Il est en revanche flagrant que ses particularités typotechnologiques avaient peu à peu été gommées pour n'en retenir que les éléments typologiques, telles les lamelles Dufour, supposés l'intégrer à la grande famille aurignacienne, et cela par opposition aux industries dites de transition, sans cesse scindées régionalement et plus ou moins inconsciemment enclavées. En outre et surtout, ces industries originellement attribuées à un Protoaurignacien sont indéniablement «oubliées» dans les grands travaux de synthèse des années quatre-vingt et quatre-vingt-dix, puisqu'on ne retenait alors de l'Aurignacien qu'une image simplifiée - et ô combien homogène - faite de grattoirs carénés, de lames robustes retouchées latéralement, de pointes de sagaie à base fendue et d'éléments d'ornement et d'art mobilier (voir p. e. Mellars, 1989 et 2004). Actuellement, l'existence de deux traditions clairement distinctes repose moins sur leur ancrage géographique encore partiellement différent que sur leurs organisations techniques et symboliques différentes.

En ce qui concerne les industries lithiques, il est maintenant admis que les productions de l'Aurignacien ancien se caractérisent par une dissociation des productions de lames d'une part et de lamelles d'autre part (voir p. e. Chadelle, 1990; Tixier, 1991; Le Brun-Ricalens, 1993; Chiotti, 1999; Teyssandier, 2000 et 2007; Bon, 2002; Ortega Cordellat, 2005; Bordes, 2006). Les lames, plutôt larges et épaisses, sont produites à partir de nucléus prismatiques unipolaires tandis que les lamelles sont en grande partie obtenues indépendamment à partir de nucléus carénés. En revanche, dans le Protoaurignacien, lames et lamelles sont d'un point de vue morphométrique nettement différentes de celles de l'Aurignacien ancien et produites majoritairement en continuité au fil d'une même chaîne technique ${ }^{2}$ (voir p. e. Bazile et Sicard, 1999; Bon, 2002; Bon et Bodu, 2002; Bordes, 2002 et 2006; Teyssandier, 2003 et 2007; Broglio et al., 2005; Maíllo Fernández, 2005; Normand et Turq, 2005; Ortega-Cobos et al., 2005). Ces deux entités diffèrent également en ce que les productions de l'Aurignacien ancien livrent d'importants corpus en matières dures animales (Liolios, 1999 et 2006) assortis de parures engageant des matières d'œuvre diversifiées (White, 1993; Vanhaeren, 2002), alors que le Protoaurignacien demeure pauvre en industrie osseuse et fournit principalement des parures en coquillage (voir p. e. Kuhn et Stiner, 1998; Kuhn et al., 2001; Vanhaeren, 2002; Vanhaeren et d'Errico, 2006). Ces différences sont encore plus claires si l'on regarde la nature des corpus en matières dures animales, nettement plus abondants et diversifiés dans les ensembles Aurignacien ancien (Liolios, 1999). De plus, ces derniers se caractérisent par des ensembles comprenant une forte proportion d'armatures de projectile (pointes à base fendue) et organisés par une stricte économie des matières premières. À ce titre, les os du squelette interne sont principalement dédiés à un outillage domestique, les bois de Renne aux pointes à base fendue et l'ivoire à des éléments de parure ou d'art mobilier (Liolios, 1999 et 2004; Teyssandier et 
Liolios, 2003; Tartar et al., 2006). Si des outillages en matières dures animales sont présents dans des ensembles protoaurignaciens, ils sont nettement moins abondants et ne semblent pas régis par une telle organisation techno-économique.

Ces différences de structure des ensembles archéologiques, pour ne pas parler de différenciation, nous ont conduits à poser la question des relations phylétiques entre ces deux entités (Teyssandier, 2003, 2006 et 2007). Comme hypothèse de départ, nous avions choisi d'y répondre en les distinguant assez radicalement (Teyssandier, 2003). Cela était déterminé à la fois par les données alors disponibles, mais aussi par une volonté de rendre à ces industries la place respective qui leur incombait dans les débats. En définitive, il est encore un peu prématuré d'interpréter la distinction Protoaurignacien/ Aurignacien ancien, mais les conceptions actuelles pourraient trouver des registres de filiation entre ces deux complexes (Bon, 2006; Bordes, 2006; Zilhão, 2006; Teyssandier, 2007). Il s'agit là d'un point important des recherches en cours, dont les premiers résultats permettent d'ores et déjà d'extraire certaines leçons.

\section{L'apparition graduelle des éléments constitutifs de l'Aurignacien}

En tout état de cause, il apparaît que la plupart des synthèses produites ont peu à peu nivelé la chronologie interne de l'Aurignacien et gommé sa variabilité pour n'en retenir que ce qui le lie à ce qui suivra et le différencie de ce qui le précéda. Or, si les différents découpages que nous sommes désormais en mesure d'opérer sont respectés, il devient possible d'affirmer que les principaux termes de la définition voyant dans l'Aurignacien la culture-mère du Paléolithique supérieur apparaissent non simultanément mais de manière plus graduelle. Cette idée se vérifie sur la base des différents matériaux qui constituent l'identité aurignacienne.

Ainsi, si les productions lithiques aurignaciennes sont traversées par une série de mutations importantes, cellesci n'apparaissent pas de manière simultanée mais, au contraire, connaissent une nette évolution au cours du temps. Les lamelles en sont un bon exemple, puisque leur systématisation constitue vraisemblablement l'une des innovations les plus sensibles des groupes aurignaciens, potentiellement motivée par la volonté de produire des supports légers pour armer des traits (Bon, 2005; Teyssandier, 2007, sous presse a et b). Or, si la production de lamelles constitue un objectif dominant des industries protoaurignaciennes, elles sont alors totalement intégrées au débitage de lames en une seule et même chaîne opératoire. Le passage à l'Aurignacien ancien marque la disjonction qui s'opère entre les lames et les lamelles, qui sont désormais produites à partir de nucléus différents. Cette disjonction opératoire s'accompagne d'une nette différenciation morphologique des objets recherchés : les lames sont longues, larges et épaisses, les lamelles courtes, étroites et minces. On pourrait donc proposer l'idée d'un cycle évolutif de leptolithisation, pour reprendre un concept cher à G. Laplace, qui s'articulerait sur la production d'éléments de projectile lithique et verrait peu à peu les sphères laminaires et lamellaires s'individualiser. L'une des nouveautés perceptible à l'Aurignacien ancien pourrait ainsi renvoyer à l'individualisation de deux grands registres d'activités, opposant caricaturalement les armes de chasse aux outillages dits domestiques (Tartar et al., 2006; Teyssandier, sous presse a et b).

Une même évolution graduelle pourrait caractériser l'essor des productions en matières dures animales, qui constituent l'une des principales innovations de l'Aurignacien. Si la généralisation du travail des matières dures animales lors de cet épisode a souvent appuyé l'idée de sa rupture avec le Paléolithique moyen, c'est à l'Aurignacien ancien qu'il prend son essor et se systématise définitivement. Parmi les types d'objets investis, la pointe de sagaie en bois de Renne reste emblématique et la base fendue, l'archétype de nos représentations de l'Aurignacien. Se dégage ici l'idée d'un système d'emmanchement et d'une pointe de trait particuliers, ayant principalement diffusé en Europe occidentale et centrale sous des formes parfois différentes selon les régions et/ou les sites considérés (Liolios, 1999 et 2006). En outre, avec l'Aurignacien ancien, ces productions sont organisées selon des règles strictes attribuant à chaque matériau exploité une destination fonctionnelle spécifique. La mise en évidence d'une phase antérieure à l'Aurignacien ancien pose la question de l'émergence des productions en matières dures animales au tout début du Paléolithique supérieur. Il est intéressant de relever que ces mêmes productions semblent plus rares en contexte Protoaurignacien et donnent principalement lieu à la réalisation d'objets tels que des poinçons et des pointes. Dans tous les sites protoaurignaciens connus, ces productions ne semblent pas encore systématisées et hiérarchisées à la mesure de ce qu'elles vont devenir à l'Aurignacien ancien (Liolios, 2006). Ces données ne vont pas sans rappeler certaines industries dites de transition où le travail des matières osseuses émerge, sans pour autant donner lieu à des productions abondantes et aussi économiquement structurées que celles de l'Aurignacien ancien (d'Errico et al., 1998). Même en contexte Aurignacien ancien, il nous semble que certaines données vont dans le sens d'une évolution technique plus graduelle. Ainsi, l'ensemble III de Geissenklösterle, pleinement aurignacien ancien du point de vue de ses productions lithiques, est totalement dépourvu de pointes à base fendue et ne présente encore qu'une industrie en matières dures animales relativement pauvre. Le système lithique aurignacien ancien aurait donc pu se mettre en place avant la pleine systématisation des industries osseuses.

Enfin, la sphère symbolique fournit également des arguments comparables puisque si la parure existe dans le Protoaurignacien, comme auparavant dans certaines industries dites de transition, elle se compose dans sa grande majorité de coquillages perforés (Taborin, 1993; Kuhn et Stiner, 1998; Kuhn et al., 2001; Vanhaeren, 2002; Vanhaeren et d'Errico, 2006; Zilhão, 2007). Le Protoaurignacien ne constitue pas à ce titre une nouveauté, puisque des parures engageant des savoir-faire complexes et des matériaux plus diversifiés sont déjà 
en place dans le Châtelperronien ou dans d'autres entités du Paléolithique supérieur initial au Proche-Orient (d'Errico et al., 1998; Kuhn et al., 2001). C'est à l'Aurignacien ancien que des matériaux plus diversifiés vont être engagés pour la fabrication d'ornements : les coquillages sont toujours présents mais s'y associent en grand nombre des dents animales ou plus rarement humaines et, plus encore, de l'ivoire et de la pierre (Vanhaeren, ibid.; White, 2002; Henry-Gambier et White, 2006; Vanhaeren et d'Errico, ibid.). Pour finir, et même si le sujet demanderait des développements qui ne trouvent pas leur place dans cet article, l'émergence de l'art figuratif appuie également l'idée d'une apparition graduelle des éléments constitutifs de l'Aurignacien. La position stratigraphique des statuettes en ivoire dans les sites souabes de Geissenklösterle et Hohle Fels montre qu'elles n'apparaissent que dans la seconde moitié de la séquence aurignacienne (Conard, 2003; Teyssandier, 2007; Zilhão, 2007), établissant un parallèle chronostylistique avec les datations directes situant certains panneaux de la grotte Chauvet autour de 31000 BP (Valladas et al., 2005) ${ }^{3}$.

Ces éléments renforcent l'idée d'une apparition non simultanée, voire non intégrée, des différents traits fondateurs de l'identité aurignacienne. À ce titre, ils sont en contradiction avec la théorie strictement migrationniste du remplacement, faisant de l'Aurignacien la culture portant d'emblée en elle toutes les modifications des débuts du Paléolithique supérieur. P. Mellars ne définissait-il pas dans un article récent l'Aurignacien de la manière suivante : «Significantly, the Aurignacian period shows an apparently sudden flowering of all the most distinctive of fully 'modern' (or, in archaeological terms, Upper Palaeolithic) cultural behaviour. Such features include: the first complex and carefully shaped bone, antler and ivory tools; a sudden proliferation of perforated animal teeth, far-travelled marine shells, carefully shaped stone and ivory beads and other forms of personal ornaments; and (at least in sites in central and western Europe) remarkably varied and sophisticated forms of both abstract and figurative art, ranging from engraved outlines of animals, to representations of both male and female sex organs, to the remarkable ivory statuettes of animal and human figures from southern Germany and the elaborate cave paintings of Chauvet cave in southeastern France $»^{4}$ (Mellars, 2004, p. 461)? Les nouvelles interprétations esquissées témoignent, au contraire, que ces différents éléments ne se sont pas développés de manière intégrée lors de l'arrivée d'un nouveau type humain. À ce titre, il devient désormais nécessaire de s'interroger sur la profondeur historique et temporelle des mutations qui vont effectivement se concrétiser, peu à peu, avec l'émergence puis le développement de l'Aurignacien.

\section{À PROPOS DES ORIGINES ET DE LA DIFFUSION DE L'AURIGNACIEN}

La remise en cause d'industries dites préaurignaciennes et l'extension géographique grandissante des ensembles rapportés au Protoaurignacien, désormais reconnus en Aquitaine (Bordes, 2006), en Bourgogne (Bon et Bodu, 2002) mais aussi en Europe centrale (Zilhão, 2006; Teyssandier, 2007) et dans les Balkans (Tsanova, 2006) modifient profondément notre perception des origines de l'Aurignacien et, plus largement, du Paléolithique supérieur européen. Si la question des origines de l'Aurignacien est encore loin d'être résolue, de premières hypothèses voient dans les ensembles de l'Ahmarien ancien une forme ancestrale des concepts forts que l'on retrouve dans le Protoaurignacien (Bar-Yosef, 2003; Bon, 2006; Teyssandier, 2006 et 2007; Tsanova, 2006; Zilhão, 2006) : débitage laminaire au percuteur tendre orienté vers la production de lames légères et de grandes lamelles de profil rectiligne, transformation en pointes d'El Wad typologiquement et stylistiquement proches des pointes dites de Font-Yves.

En revanche, l'Aurignacien ancien qui fait stratigraphiquement suite au Protoaurignacien en Europe occidentale (voir p. e. Bordes, 2006; Normand, 2006; Zilhão, 2006; Arrizabalaga et al., 2007; Teyssandier, 2007) semble, lui, au départ, un phénomène d'origine et d'essence européenne relativement circonscrit dans l'espace aux régions aquitano-pyrénéennes et au SudOuest de l'Allemagne.

Le Protoaurignacien apparaît donc comme la première industrie du Paléolithique supérieur à l'échelle du continent européen. Son ancrage dans des contextes géographiques et environnementaux variés montre que les solutions techniques adoptées ont transcendé des écosystèmes diversifiés. Enfin, sa reconnaissance vient un peu atténuer le sentiment de rupture radicale avec les complexes précédents qui prévalait dans les interprétations anciennes (Bordes, 2006). Ces nouvelles données nous obligent à reconsidérer les dynamiques évolutives à l'œuvre lors de l'émergence du Paléolithique supérieur européen.

\section{Un auteur incertain}

L'association de l'Aurignacien à la première expansion d'Homo sapiens sapiens en Europe pourrait être entendue comme un fait qui ne se discute pas. Il en est ainsi depuis plusieurs décennies (voir par exemple Breuil, 1913) et même la retentissante découverte à Saint-Césaire de restes néandertaliens en contexte châtelperronien (Lévêque et Vandermeersch, 1980) n'est pas venue ébranler l'édifice. Pourtant, il faut convenir que la situation reste très incertaine et que les données anthropologiques ne sont pas nombreuses sur le sujet. Il n'existe aucun contexte archéologique clair ayant livré des restes humains indubitablement «modernes » associés au Protoaurignacien ou à l'Aurignacien ancien. L'un des derniers gisements qui permettaient de soutenir cette hypothèse, la grotte du Vogelherd dans le Jura souabe, a vu ses restes d'hommes anatomiquement modernes que l'on pensait associés aux pointes à base fendue dater d'une phase récente du Néolithique (Conard et al., 2004). D'autres vestiges humains isolés, comme ceux de Kelsterbach ou Hahnöfersand en Allemagne, jusqu'alors considérés d'âge 
paléolithique supérieur ancien, viennent d'être considérablement rajeunis (Street et al., 2006), à l'image des résultats obtenus sur les fossiles de la Rochette ou de Cro-Magnon, ces derniers s'avérant d'âge gravettien (Orschiedt, 2002; Henry-Gambier, 2002). Ailleurs, aucun des sites diagnostiqués «Protoaurignacien» ou «Aurignacien ancien» ne livre de restes humains dont l'attribution soit sans équivoque. Les discussions se poursuivent autour du cas de Brassempouy où une dizaine de vestiges sont associés à une industrie indubitablement Aurignacien ancien (Bon, 2002; HenryGambier et al., 2004). Mais ici, c'est la nature du corpus anthropologique, composé de dents isolées et de quelques fragments crâniens et post-crâniens, qui pose problème. Pour D. Henry-Gambier et al. (2004), il n'est pas possible de trancher, sur la foi de tels éléments, entre Néandertal et Homme moderne alors que S. Bailey et J.-J. Hublin assurent qu'il s'agit sans conteste d'individus devant être rangés parmi les Homo sapiens sapiens (Bailey et Hublin, 2005).

Ailleurs, les vestiges anthropologiques identifiables font défaut et nous laissent face à un acteur des débuts de l'Aurignacien encore bien invisible. Il faut toutefois mentionner les découvertes effectuées à Oase (Roumanie), qui nous assurent que l'Homme moderne était présent en Europe centrale dès 35000 BP, sans pour autant livrer d'informations sur leurs pratiques culturelles (Trinkaus et al., 2003a et b). Finalement, il faut attendre des contextes attribués à l'Aurignacien récent, autour de $31000 \mathrm{BP}$, pour rencontrer de nouveau des vestiges humains attribuables sans équivoque à l'Homme moderne (Henry-Gambier, 2007; Trinkaus, 2007). De telle sorte que la question demeure posée de savoir quels hommes occupaient l'Europe à l'aube du Paléolithique supérieur, surtout si l'on tient compte du fait que certaines données paléoanthropologiques vont dans le sens d'un degré substantiel d'hybridation entre les premiers Sapiens européens connus et les populations locales néandertaliennes (Trinkaus, 2007). Des problèmes identiques sont posés lorsque l'on cherche à définir le ou les auteurs des industries dites de transition du Paléolithique moyen au Paléolithique supérieur, interprétées dans leur ensemble comme l'œuvre des derniers Néandertaliens, alors qu'hormis le cas du Châtelperronien de Saint-Césaire (Lévêque et Vandermeersch, 1980) et d'Arcy-sur-Cure (Leroi-Gourhan, 1958; Bailey et Hublin, 2006), aucune donnée anthropologique ne permet d'étayer cette association.

\section{SURTOUT NE PAS CONCLURE...}

Les études technologiques ont en grande partie confirmé la validité des découpages établis à l'aide de la typologie, puisque le Protoaurignacien et l'Aurignacien ancien étaient déjà reconnus sur cette base dès les années soixante (voir par exemple Sonneville-Bordes, 1960, pour l'Aurignacien ancien et Laplace, 1966, pour le Protoaurignacien). Cependant, en affinant les critères d'analyse, nous sommes désormais confrontés à une plus large variabilité des productions. La difficulté réside désormais à dépasser ce stade formel et descriptif pour parvenir à intégrer cette documentation dans une vision plus synthétique, rendant compte des dynamiques évolutives à l'œuvre à l'orée du Paléolithique supérieur. C'est à ce stade de l'analyse que les représentations passées peuvent devenir un obstacle à notre réflexion. En effet, il est inutile de décrire une variabilité de plus en plus importante des productions lithiques si elles restent enfermées dans des classifications générales qui en surdéterminent l'interprétation.

Une piste de recherche fondamentale dans les années à venir consistera à poser la question des relations entre Protoaurignacien et Aurignacien ancien. Ce faisant, c'est à la définition même de l'Aurignacien que l'on touche et, en définitive, c'est à l'usage que l'on fait de ces termes que nous sommes renvoyés. Nous serions personnellement enclins à les considérer comme un outil de travail, des sortes d'idéaux types qui n'ont d'existence que méthodologique et comparative. Ils sont voués à évoluer avec les données et ont pour seule fin de fixer des critères provisoires de comparaison entre des ensembles. Or, la plupart du temps, alors que nous avons tous conscience de la valeur strictement méthodologique de nos grilles de lecture culturelles, les ensembles composés deviennent des réalités si incarnées qu'il nous devient même difficile de les réajuster à partir de nos données.

Ainsi, si l'Aurignacien «vrai» doit intégrer tous les éléments classiques de sa définition (débitage de lames et de lamelles, systématisation du travail des matières dures animales, parures diversifiées, art...), alors l'Aurignacien pourrait tout autant ne renvoyer qu'à un événement culturel proprement européen, celui de l'Aurignacien ancien (I), faciès princeps de sa définition. Les données ne semblent pas totalement aller en ce sens, car des filiations évolutives sont pressenties entre Protoaurignacien et Aurignacien ancien. Il semble notamment que, parmi d'autres, la recherche de solutions techniques pour armer des traits de projectile lithique pourrait constituer l'un des moteurs de l'évolution technologique traversant les débuts de l'Aurignacien. Cette recherche serait perceptible avant l'émergence de l'Aurignacien, comme semblent en témoigner l'allégement des productions lithiques et la recherche de supports convergents dans différents complexes dits de transition (Émiréen, Bachokirien, Bohunicien, etc.). In fine, une certaine arythmie présiderait à la mise en place des caractères constitutifs du Paléolithique supérieur (Teyssandier, 2007, sous presse a et b).

Les limites auxquelles nous sommes confrontés dans notre recherche ordinaire ne sont donc pas seulement composées de limites propres aux données, toujours partielles, aux problèmes d'échelles chronologiques et géographiques qui rendent difficiles les reconstitutions dynamiques; elles sont aussi le fait de choix épistémologiques historiques. Pour accéder au statut de science, la préhistoire devait se doter impérativement d'un objet scientifique qui soit concret, positif, et cela en utilisant nécessairement les méthodes propres aux sciences de la nature. Cela a eu entre autres conséquences l'enracinement de nos modèles dans la pensée évolutionniste et la réification des concepts utilisés. Notre conception de l'Aurignacien est tout à fait symptomatique de ce 
fait. L'apparition de questionnements d'ordre anthropologique suppose pourtant des réajustements épistémologiques, mais notre pensée reste écartelée entre ces modèles anciens, efficaces et fondateurs, et la nécessité d'en concevoir de nouveaux. C'est bien là que se situe l'enjeu des recherches futures, non pas dans le seul progrès de nos outils d'analyse, aussi indéniable sur le terrain qu'en laboratoire, mais bel et bien dans la refonte de certains paradigmes. Si les recherches sur l'Aurignacien ne sont là que pour rendre compte que ce technocomplexe est bien la marque de la diffusion des premiers hommes modernes en Europe, alors la variabilité industrielle décrite sur le terrain demeurera vaine au profit d'idées toutes théoriques qui ne sont par ailleurs pas exemptes d'une idéologie finaliste.

Remerciements : Nous remercions sincèrement F. Bon pour sa relecture attentive du manuscrit. Des discussions avec S. Dubois, D. Henry-Gambier, V. Guillomet-Malmassari, L.G. Straus et J. Zilhão ont été précieuses pour développer notre réflexion sur les éléments évoqués dans cet article. Que tous soient ici sincèrement remerciés.

\section{NOTES}

(1) Cette vision fait écho à l'analyse menée par Marcellin Boule dans les mêmes années sur l'anatomie et le statut des Néandertaliens, à la suite de sa description du fossile de la Chapelle-aux-Saints découvert en 1908
(Boule, 1911 et 1913). M. Boule y dépeint un être proche des singes anthropoïdes mais assurément humain dont l'anatomie robuste et «brutale» révèlerait «l'absence probable de toutes traces de préoccupation d'ordre esthétique ou d'ordre moral » et «la prédominance des fonctions purement végétatives ou bestiales» (Boule, 1913 et voir également Lumley et Hurel, 2006).

(2) Il importe toutefois de signaler que dans l'ensemble des séries rapportées au Protoaurignacien, des productions lamellaires autonomes sont attestées. La question qui est posée est davantage celle de l'indépendance des lames vis-à-vis des lamelles que l'inverse.

(3) Il a été récemment proposé qu'un art figuratif se développerait également dans le Protoaurignacien, suite à la découverte de fragments rocheux effondrés de la paroi et décorés d'ocre rouge à Fumane (Broglio dir., 2005 ; Broglio et al., 2007). Notons cependant que tous les fragments en question ont été découverts au sommet de la séquence (niveaux D3D6) dont les dates se situent autour de 32-31000 BP. Ainsi, si l'industrie lithique associée possède des caractères indubitablement protoaurignaciens, les datations relevées tendent à lui conférer un âge assez récent, tout du moins pour le sommet de la séquence aurignacienne. Se pose ici la question de la perduration possible d'industries lithiques de type Protoaurignacien au-delà des frontières chronologiques traditionnellement assignées à l'Aurignacien ancien.

(4) «De manière significative, l’Aurignacien montre un développement soudain de toutes les caractéristiques distinctives d'un comportement «totalement» moderne (ou, en termes archéologiques, de type Paléolithique supérieur). Ces caractéristiques incluent : les premiers outils complexes et soigneusement façonnés en os, bois de Renne et ivoire; une soudaine prolifération de dents animales perforées, de coquillages marins de provenance lointaine, de perles soigneusement façonnées en pierre et en ivoire et d'autres formes de parures; et (au moins dans les sites d'Europe centrale et occidentale), des formes remarquablement variées et sophistiquées d'art tant abstrait que figuratif - allant de gravures d'animaux à des représentations d'organes sexuels masculins et féminins jusqu'aux remarquables statuettes figuratives animales et humaines en ivoire d'Allemagne du Sud ou aux peintures pariétales élaborées de la grotte Chauvet en France du Sud-Est» (Mellars, 2004, p. 461, traduction des auteurs).

\section{RÉFÉRENCES BIBLIOGRAPHIQUES}

ADAMS B., RINGER A. (2004) - New ${ }^{14} \mathrm{C}$ dates suggest more recent age for Hungarian Early Upper Palaeolithic sites, Current Anthropology, vol. 45, p. 541-551.

ARRIZABALAGA A., BON F., MAÍLLO FERNÁNDEZ J. M., NORMAND C., ORTEGA I. (2007) - Territoires et frontières de 1'Aurignacien dans les Pyrénées occidentales et les Cantabres, in N. Cazals, J. González Urquijo et X. Terradas dir., Fronteras naturales y fronteras culturales en los Pireneos préhistoricos, Universidad de Cantabria, Santander, p. 301-318.

BACHELARD G. (1934) - La formation de l'esprit scientifique. Contribution à une psychanalyse de la connaissance objective, Librairie philosophique J. Vrin, Paris, 5 édition, 1967, 257 p.

BAR-YOSEF O. (2003) - Away from home: prehistoric colonizations, exchanges and diffusions in the Mediterranean basin, in B. Vandermeersch dir., Échanges et diffusion dans la Préhistoire méditerranéenne, CTHS, Paris, p. 71-81.

BAILEY S.E., HUBLIN J.-J. (2005) - Who made the early Aurignacian? A reconsideration of the Brassempouy dental remains, Bulletins et Mémoires de la Société d'Anthropologie de Paris, t. 17, 1-2, p. 115121.

BAILEY S.E., HUBLIN J.-J. (2006) - Dental remains from the Grotte du Renne at Arcy-sur-Cure (Yonne), Journal of Human Evolution, vol. 50, p. 485-508

BAZILE F., SICARD S. (1999) - Le premier Aurignacien du Languedoc oriental dans son contexte méditerranéen, in D. Sacchi dir., Les faciès leptolithiques du Nord-Ouest méditerranéen : milieux naturels et culturels, XXIV congrès préhistorique de France, Carcassonne, 1994 Société préhistorique française, Paris, p. 117-125.

BON F. (2002) - L'Aurignacien entre mer et océan. Réflexion sur l'unité des phases anciennes de l'Aurignacien dans le Sud de la France, Mémoire 29, Société préhistorique française, Paris.
BON F. (2005) - Little big tool. Enquête autour du succès de la lamelle, in F. Le Brun-Ricalens dir., Productions lamellaires attribuées à l'Aurignacien, chaînes opératoires et perspectives technoculturelles, ArchéoLogiques, 1, MNHA, Luxembourg, p. 479-484.

BON F. (2006) - A brief overview of Aurignacian cultures in the context of the industries of the transition from the Middle to the Upper Palaeolithic, in O. Bar-Yosef et J. Zilhão dir., Towards a definition of the Aurignacian, Instituto Português de Arqueologia, Lisbonne, p. 133144.

BON F., BODU P. (2002) - Analyse technologique du débitage Aurignacien, in $\mathrm{B}$. Schmider dir., L'Aurignacien de la grotte du Renne. Les fouilles d'André Leroi-Gourhan à Arcy-sur-Cure (Yonne), 34 suppl. à Gallia Préhistoire, éd. du CNRS, Paris, p. 115-133.

BORDES J.-G. (2002) - Les interstratifications Châtelperronien/ Aurignacien du Roc de Combe et du Piage (Lot, France). Analyse taphonomique des industries lithiques; implications archéologiques, thèse de doctorat de l'université de Bordeaux I.

BORDES J.-G. (2006) - News from the West: a reevaluation of the classical Aurignacian sequence of the Perigord, in O. Bar-Yosef et J. Zilhão dir., Towards a definition of the Aurignacian, Trabalhos de Arqueologia, 45, Instituto Português de Arqueologia, Lisbonne, p. 147-171.

BOULE M. (1911) - L'homme fossile de la Chapelle-aux-Saints, Annales de Paléontologie, vol. 6, p. 109-172.

BOULE M. (1913) - L'homme fossile de la Chapelle-aux-Saints, Annales de Paléontologie, vol. 7, p. 1-72.

BREUIL H. (1907) - L'Aurignacien présolutréen. Épilogue d'une controverse, Revue préhistorique, t. 4, p. 5-46.

BREUIL H. (1913) - Les subdivisions du Paléolithique supérieur et leur signification, Congrès international d'anthropologie et d'archéologie préhistorique, $14^{e}$ session, Genève, I, p. 165-238. 
BREUIL H. (1929) - La Préhistoire. Leçon d'ouverture de la chaire de préhistoire au Collège de France, 30 décembre 1929, Revue des cours et conférences, $31^{\mathrm{e}}$ année, $\mathrm{n}^{\circ} 2$, p. 97-113.

BROGLIO A. dir. (2005) - Pitture Aurignaziane nella Grotta di Fumane, in A. Broglio et G. Dalmeri dir., Pitture Paleolitiche nelle Prealpi Venete. Grotta di Fumane e Riparo Dalmeri, Preistoria Alpina, ${ }^{\circ}$ spécial, Memorie del Museo Civico di Storia Naturale du Verona, Vérone, p. 11-63.

BROGLIO A., BERTOLA S., DE STEFANI M., MARINI D., LEMORINI C., ROSSETTI P. (2005) - La production lamellaire et les armatures lamellaires de l'Aurignacien ancien de la grotte de Fumane (monts Lessini, Vénétie), in F. Le Brun-Ricalens dir., Productions lamellaires attribués à l'Aurignacien, chaînes opératoires et perspectives technoculturelles, Archéologiques, 1, Musée national d'histoire et d'art, Luxembourg, p. 415-436.

BROGLIO A., GIACHI G., GURIOLI F., PALLECCHI P. (2007) - Les peintures aurignaciennes de la grotte de Fumane (Italie), in H. Floss et N. Rouquerol dir., Les chemins de l'art aurignacien en Europe, éd. Musée-Forum Aurignac, Aurignac, p. 157-170.

CABRERA VALDÈS V., MAILLO J. M., LLORET M., BERNALDO DE QUIRÓS F. (2001) - La transition vers le Paléolithique supérieur dans la grotte du Castillo (Cantabrie, Espagne) : la couche 18, L'Anthropologie, t. 105, p. 505-532.

CHADELLE J.-P. (1990) - Le site de plein air de Corbiac-Vignoble à Bergerac (Dordogne). Technologie lithique et mode d'occupation, Le silex de sa genèse à l'outil, Cahiers du Quaternaire, 17, éd. du CNRS, Paris, p. 385-390.

CHIOTTI L. (1999) - Les industries lithiques des niveaux aurignaciens de l'abri Pataud, Les Eyzies-de-Tayac (Dordogne) : étude technologique et typologique, thèse de doctorat du Muséum national d'histoire naturelle, Institut de paléontologie humaine, 2 vol.

CONARD N.J. (2003) - Palaeolithic ivory sculptures from Southwestern Germany and the origins of figurative art, Nature, vol. 426, p. 830-832.

CONARD N.J., BOLUS M. (2003) - Radiocarbon dating the appearance of modern humans and timing of cultural innovations in Europe: new results and new challenges, Journal of Human Evolution, vol. 44, p. 331-371.

CONARD N.J., GROOTES P.M., SMITH F.H. (2004) - Unexpectedly recent dates for human remains from Vogelherd, Nature, vol. 430, p. 198-201.

COYE N. (1997) - La préhistoire en parole et en acte. Méthodes et enjeux de la pratique archéologique (1830-1950), éd. L'Harmattan, Paris, 338 p.

DEMARS P.-Y., HUBLIN J.-J. (1989) - La transition Néandertaliens/ Hommes de type moderne en Europe occidentale : aspects paléontologiques et culturels, in B. Vandermeersch dir., L'Homme de Neandertal. L'extinction, ERAUL, t. 34, Liège, p. 23-37.

D'ERRICO F., ZILHÃO J., JULIEN M., BAFFIER D., PELEGRIN J. (1998) - Neanderthal acculturation in Western Europe? A critical review of the evidence and its interpretation, Current Anthropology, vol. 39, p. S1-S44.

DUBOIS S., BON F. (2006) - Henri Breuil et les origines de la «bataille aurignacienne», Sur les chemins de la Préhistoire. L'abbé Breuil du Périgord à l'Afrique du Sud, catalogue de l'exposition présentée au musée d'art et d'histoire Louis-Senlecq de L'Isle-Adam, Somogy, Paris, p. 135-147.

GORING-MORRIS N., BELFER-COHEN A. dir. (2003) - More than meets the eye, Oxbow Books, Oxford.

GUILLOMET-MALMASSARI V. (2006) - Du simple et du complexe un rapport constructif de la préhistoire (XIX ${ }^{\mathrm{e}}-\mathrm{XX}^{\mathrm{e}}$ siècles), in L. Astruc, F. Bon, V. Léa, P.-Y. Milcent et S. Philibert dir., Normes techniques et pratiques sociales, de la simplicité des outillages pré- et protohistoriques, $X X V I^{e s}$ rencontres internationales d'archéologie et d'histoire d'Antibes, ADPCA, Antibes, p. 15-24.

HAESAERTS P., TEYSSANDIER N. (2003) - The Early Upper Palaeolithic occupation of Willendorf II (Lower Austria): a contribution to the chronostratigraphic and cultural context of the beginning of the Upper Palaeolithic in Central Europe, in J. Zilhão et F. d'Errico dir., The chronology of the Aurignacian and of the transitional technocomplexes. Dating, stratigraphies, cultural implications, Trabalhos de Arqueologia, 33, Instituto Português de Arqueologia, Lisbonne, p. $133-151$

HENRY-GAMBIER D. (2002) - Les fossiles de Cro-Magnon (Les Eyzies-de-Tayac, Dordogne) : nouvelles données sur leur position chronologique et leur attribution culturelle, Paléo, t. 14, p. 201204.

HENRY-GAMBIER D. (2007) - Le peuplement aurignacien de l'Europe, in $\mathrm{H}$. Floss et $\mathrm{N}$. Rouquerol dir., Les chemins de l'art aurignacien en Europe, éd. Musée-Forum Aurignac, Aurignac, p. 105-116.

HENRY-GAMBIER D., WHITE R. (2006) - Modifications artificielles des vestiges humains de l'Aurignacien ancien de la grotte des Hyènes (Brassempouy, Landes). Quelle signification?, in V. Cabrera Valdés, F. Bernaldo de Quirós et J. M. Maíllo Fernández dir., En el centenario de la Cueva de El Castillo : el ocaso de los neandertales, UNED, Madrid, p. 71-88.

HENRY-GAMBIER D., MAUREILLE B., WHITE R. (2004) - Vestiges humains des niveaux de l'Aurignacien ancien du site de Brassempouy (Landes), Bulletins et Mémoires de la Société d'Anthropologie de Paris, t. 16, n 1-2, p. 49-87.

KOZLOWSKI J.K. (1993) - L'Aurignacien en Europe et au ProcheOrient, in L. Bánesz et J.K. Kozlowski dir., Aurignacien en Europe et au Proche-Orient, Institut archéologique de l'Académie slovaque des sciences, Bratislava,p. 283-291.

KOZLOWSKI J.K. (2004) - Early Upper Palaeolithic Levallois-derived industries in the Balkans and in the Middle Danube basin, Anthropologie, vol. XLII, 3, p. 263-280.

KOZLOWSKI J.K., OTTE M. (2000) - The formation of the Aurignacian in Europe, Journal of Anthropological Research, vol. 56, p. 513534.

KUHN S.L., STINER M.C. (1998) - The earliest Aurignacian of Riparo Mochi (Liguria, Italy), Current Anthropology, vol. 39, p. 175-189.

KUHN S.L., STINER M.C., REESE D.S., GÜLEC E. (2001) - Ornaments of the earliest Upper Palaeolithic: new insights from the Levant, Proceedings of the National Academy of Sciences of the USA, vol. 98, p. 7641-7646.

LAPLACE G. (1966) - Recherches sur l'origine et l'évolution des complexes leptolithiques, Mélanges d'Archéologie et d'Histoire, École française de Rome, $4^{\mathrm{e}}$ suppl., éd. de Boccard, Paris.

LE BRUN-RICALENS F. (1993) - Réflexions préliminaires sur le comportement litho-technologique et l'occupation du territoire du pays des Serres à l'Aurignacien : le gisement de «Toulousète» à Beauville (Lot-et-Garonne), une occupation moustérienne et aurignacienne de plein air, Paléo, t. 5, p. 127-153.

LEROI-GOURHAN A. (1958) - Étude des restes humains fossiles provenant des grottes d'Arcy-sur-Cure, Annales de Paléontologie, vol. 44 , p. $87-148$.

LÉVÊQUE F., VANDERMEERSCH B. (1980) - Découverte de restes humains dans un niveau castelperronien à Saint-Césaire (CharenteMaritime), Comptes rendus de l'Académie des sciences de Paris, vol. 291, p. 187-189.

LIOLIOS D. (1999) - Variabilité et caractéristiques du travail des matières osseuses au début de l'Aurignacien : approche technologique et économique, thèse de doctorat de l'université Paris X-Nanterre.

LIOLIOS D. (2004) - Le travail des matières osseuses au début de l'Aurignacien : aspects techniques, économiques et symboliques de l'organisation de la production de Geissenklösterle (Jura souabe), in P. Bodu et C. Constantin dir., Approches fonctionnelles en préhistoire Société préhistorique française, Paris, p. 371-386.

LIOLIOS D. (2006) - Reflections on the role of bone tools in the definition of the Early Aurignacian, in O. Bar-Yosef et J. Zilhão dir. Towards a definition of the Aurignacian, Trabalhos de Arqueologia, 45, Instituto Portuguese de Arqueologia, Lisbonne, p. 37-51. 
LUMLEY M.-A. de., HUREL A. (2006) - La paléoanthropologie dans les Annales de Paléontologie sous l'impulsion de Marcellin Boule et Jean Piveteau, Annales de Paléontologie, vol. 92, p. 217-222.

MAÍLLO FERNÁNDEZ J. M. (2005) - La production lamellaire de l'Aurignacien de la grotte Morín (Cantabrie, Espagne), in F. Le BrunRicalens dir., Productions lamellaires attribués à l'Aurignacien, chaînes opératoires et perspectives technoculturelles, Archéologiques, 1, Musée national d'histoire et d'art, Luxembourg, p. 339-357.

MELLARS P. (1989) - Major issues in the emergence of modern humans, Current Anthropology, vol. 30, p. 349-385.

MELLARS P. (2004) - Neanderthals and the modern human colonization of Europe, Nature, vol. 432, p. 461-465.

MORTILLET G. de (1872) - Classification des diverses périodes de l'Âge de la Pierre, Congrès international d'archéologie et d'anthropologie préhistoriques, Bruxelles.

MORTILLET G. de (1885) - Le Préhistorique, antiquité de l'homme, éd. C. Reinwald, Paris.

NORMAND C., TURQ A. (2005) - L'Aurignacien de la grotte d'Isturitz (France) : la production lamellaire dans la séquence de la salle de Saint-Martin, in F. Le Brun-Ricalens dir., Productions lamellaires attribués à l'Aurignacien, chaînes opératoires et perspectives technoculturelles, Archéologiques, 1, Musée national d'histoire et d'art Luxembourg, p. 373-392.

NORMAND C. (2006) - L'Aurignacien de la salle de Saint-Martin (grotte d'Isturitz, commune de Saint-Martin-d'Arberoue, PyrénéesAtlantiques) : données préliminaires sur l'industrie lithique recueillie lors des campagnes 2000-2002, in F. Bon, J. M. Maíllo Fernández et D. Ortega i Cobos dir., Autour des concepts de Protoaurignacien, d'Aurignacien archaïque, initial et ancien. Unité et variabilité des comportements techniques des premiers groupes d'hommes modernes dans le Sud de la France et le Nord de l'Espagne, Prehistoria y Arqueologia, Serie I, t. 15, UNED, Madrid, p. 145-174.

OLIVA M. (1989) - The early Aurignacian industry from Vedrovice II (Southern Moravia) and the question of the Aurignacian origins, Anthropologie, vol. 27, p. 251-264.

ORSCHIEDT J. (2002) - Datation d'un vestige humain provenant de la Rochette (Saint-Léon-sur-Vézère, Dordogne) par la méthode du carbone 14 en spectrométrie de masse, Paléo, t. 14, p. 239-240.

ORTEGA I COBOS D., SOLER MASFERRER N., MAROTO GENOVER J. (2005) - La production des lamelles pendant l'Aurignacien archaïque dans la grotte de l'Arbreda : organisation de la production, variabilité des méthodes et des objectifs, in F. Le Brun-Ricalens dir., Productions lamellaires attribués à l'Aurignacien, chaînes opératoires et perspectives technoculturelles, Archéologiques, 1, Musée national d'histoire et d'art, Luxembourg, p. 359-373.

ORTEGA CORDELLAT I. (2005) - La production lamellaire du niveau Aurignacien de Barbas III (Creysse, Dordogne), in F. Le BrunRicalens dir., Productions lamellaires attribués à l'Aurignacien, chaînes opératoires et perspectives technoculturelles, Archéologiques, 1, Musée national d'histoire et d'art, Luxembourg, p. 211-224.

RIGAUD J.-P. (2001) - À propos de la contemporanéité du Castelperronien et de l'Aurignacien dans le nord-est de l'Aquitaine : une révision des données et ses implications, in J. Zilhão, T. Aubry et A. F. Carvalho dir., Les premiers hommes modernes de la péninsule Ibérique, Trabalhos de Arqueologia, 17, Instituto Português de Arqueologia, Lisbonne, p. 61-68.

RIGAUD J.-P., LUCAS G. (2006) - The first Aurignacian technocomplexes in Europe: a revision of the Bachokirian, in O. Bar-Yosef et J. Zilhão dir., Towards a definition of the Aurignacian, Trabalhos de Arqueologia, 45, Instituto Português de Arqueologia, Lisbonne, p. 277-284.

SCHMIDT R.R. (1912) - Die diluviale Vorzeit Deutschlands, E. Schweizerbartsche Verlagsbuchhandlung, Stuttgart.

SONNEVILLE-BORDES D. de (1960) - Le Paléolithique supérieur en Périgord, éd. Delmas, Bordeaux, 2 vol.

STRAUS L.G. (1996) - Continuity or rupture; convergence or invasion; adaptation or catastrophe; mosaic or monolith: views on the Middle to Upper Palaeolithic transition in Iberia, in E. Carbonell et M. Vaquero dir., The last Neandertals, the first Anatomically Modern Humans: a tale about the human diversity. Cultural change and human evolution: the crisis at $40 \mathrm{ka} \mathrm{BP}$, Universitat Rovira i Virgili, Tarragona, p. 203-218.

STRAUS L.G. (2003) - "The Aurignacian"? Some thoughts, in J. Zilhão et F. d'Errico dir., The chronology of the Aurignacian and of the transitional technocomplexes. Dating, stratigraphies, cultural implications, Trabalhos de Arqueologia, 33, Instituto Português de Arqueologia, Lisbonne, p. 11-17.

STREET M., TERBERGER T., ORSCHIEDT J. (2006) - A critical review of the German Palaeolithic hominin record, Journal of Human Evolution, vol. 51, p. 551-579.

TABORIN Y. (1993) - La parure en coquillage au Paléolithique, suppl. à Gallia Préhistoire, 29, éd. du CNRS, Paris.

TARTAR E., TEYSSANDIER N., BON F., LIOLIOS D. (2006) - Équipement de chasse, équipement domestique : une distinction efficace? Réflexion sur la notion d'investissement technique dans les industries aurignaciennes, in L. Astruc, F. Bon, V. Léa, P.-Y. Milcent et S. Philibert dir., Normes techniques et pratiques sociales, de la simplicité des outillages pré-et protohistoriques, XXVI ${ }^{\text {s }}$ rencontres internationales d'archéologie et d'histoire d'Antibes, ADPCA, Antibes, p. 107-118.

TEYSSANDIER N. (2000) - L'industrie lithique aurignacienne du secteur II de Barbas (Creysse, Dordogne). Analyse technique et implications archéologiques, in E. Boëda et V. Guillomet-Malmassari dir., Des comportements techniques dans la Préhistoire, Laboratoire d'ethnologie et de sociologie comparative, Atelier 20, Nanterre, p. 29-59.

TEYSSANDIER N. (2003) - Les débuts de l'Aurignacien en Europe. Discussion à partir des sites de Geissenklösterle, Willendorf II, KremsHundssteig et Bacho Kiro, thèse de doctorat de l'université de Paris $\mathrm{X}$-Nanterre.

TEYSSANDIER N. (2006) - Questioning the first Aurignacian: mono or multi cultural phenomenon during the formation of the Upper Palaeolithic in Central Europe and the Balkans, Anthropologie, vol. XLIV/1, p. 9-29.

TEYSSANDIER N. (2007) - En route vers l'ouest. Les débuts de l'Aurignacien en Europe, BAR International Series, 1638, John \& Erica Hedges Ltd., Oxford.

TEYSSANDIER N. (sous presse a) - L'émergence du Paléolithique supérieur en Europe : mutations culturelles et rythmes d'évolution, Paléo, t. 19, 2007, 24 p., 8 fig.

TEYSSANDIER N. (sous presse b) - Revolution or Evolution: the emergence of the Upper Palaeolithic in Europe, World Archaeology, vol. 40, 2008.

TEYSSANDIER N., LIOLIOS D. (2003) - Defining the earliest Aurignacian in the Swabian Alp: the relevance of the technological study of the Geissenklösterle (Baden-Württemberg, Germany) lithic and organic productions, in J. Zilhão et F. d'Errico dir., The chronology of the Aurignacian and of the transitional technocomplexes. Dating, stratigraphies, cultural implications, Trabalhos de Arqueologia, 33, Instituto Português de Arqueologia, Lisbonne, p. 179-196.

TEYSSANDIER N., BOLUS M., CONARD N.J. (2006) - The Early Aurignacian in central Europe and its place in an European perspective, in O. Bar-Yosef et J. Zilhão dir., Towards a definition of the Aurignacian, Trabalhos de Arqueologia, 45, Instituto Portuguese de Arqueologia, Lisbonne, p. 241-256.

TEYSSANDIER N., MESTER Zs., LENGYEL Gy. (en préparation) Istàllòskö revisited. Historical, technological and taphonomic analyses of a key-sequence of the Early Upper Palaeolithic in central Europe.

TIXIER J. (1991) - Et passez au pays des silex : rapportez-nous des lames !, 25 ans d'études technologiques en préhistoire, XI ${ }^{e s}$ rencontres internationales d'Archéologie et d'Histoire d'Antibes, éd. APDCA, Juan-les-Pins, p. 235-243.

TRINKAUS E. (2007) - European early modern humans and the fate of Neandertals, Proceedings of the National Academy of Sciences of the USA, vol. 104 , p. 7367-7372. 
TRINKAUS E., MOLDOVAN O., MILOTA S., BÎLGĂR A., SARCINA L. (2003a) - An early modern human from the Peştera cu Oase, Romania, Proceedings of the National Academy of Sciences of the USA, vol. 100, p. 11231-11236.

TRINKAUS E., MILOTA Ş., RODRIGO R., GHERASE M., MOLDOVAN O. (2003b) - Early modern human cranial remains from the Peştera cu Oase, Romania, Journal of Human Evolution, vol. 45, p. 245-253.

TSANOVA T. (2006) - Les débuts du Paléolithique supérieur dans l'Est des Balkans. Réflexion à partir de l'étude taphonomique et technoéconomique des ensembles lithiques des sites de Bacho Kiro (couche 11), Temnata (couches VI et 4) et Kozarnika (couche VII), thèse de doctorat de l'université Bordeaux 1.

TSANOVA T., BORDES J.-G. (2003) - Contribution au débat sur l'origine de l'Aurignacien : principaux résultats d'une étude technologique de l'industrie lithique de la couche 11 de Bacho Kiro, in T. Tsonev et E. Montagnari Kokelj dir., The humanized mineral world: towards social and symbolic evaluation of prehistoric technologies in South Eastern Europe, éd. ERAUL, Liège, p. 41-50.

VALLADAS H., TISNÉRAT-LABORDE N.. CACHIER H., KALTNECKER E., ARNOLD M., OBERLIN C., ÉVIN J. (2005) - Bilan des datations carbone 14 effectuées sur des charbons de bois de la grotte Chauvet, Bulletin de la Société préhistorique française, t. 102, $\mathrm{n}^{\circ} 1$, p. 109-113.

VALOCH K. (1990) - La Moravie il y a 40000 ans, in C. Farizy dir., Paléolithique moyen récent et Paléolithique supérieur ancien en Europe, Mémoires du musée de Préhistoire d'Île-de-France, 3, éd. APRAIF, Nemours, p. 115-124

VANHAEREN M. (2002) - Les fonctions de la parure au Paléolithique supérieur: de l'individu à l'unité culturelle, thèse de doctorat de l'université Bordeaux 1.

VANHAEREN M., D'ERRICO F. (2006) - Aurignacian ethno-linguistic geography of Europe revealed by personal ornaments, Journal of Archaeological Science, vol. 33, 8, p. 1105-1128.

WHITE R. (1993) - A social and technological view of Aurignacian and Castelperronian personal ornaments in France, in V. Cabrera Valdés dir., El origen del hombre moderno en el Suroeste de Europa, Universidad Nacional de Educación a Distancia, Madrid, p. 327357.
WHITE R. (2002) - Observations technologiques sur les objets de parure, in B. Schmider dir., L'Aurignacien de la grotte du Renne. Les fouilles d'André Leroi-Gourhan à Arcy-sur-Cure (Yonne), 34e suppl. à Gallia Préhistoire, éd. du CNRS, Paris, p. 257-266.

ZILHÃO J. (2006) - Neandertals and Moderns mixed, and it matters, Evolutionary Anthropology, vol. 15, p. 183-195.

ZILHÃO J. (2007) - The emergence of ornaments and art: an archaeological perspective on the origins of "behavioral modernity", Journal of Archaeological Research, vol. 15, p. 1-54.

ZILHÃO J., d'ERRICO F. (1999) - The chronology and taphonomy of the Earliest Aurignacian and its implications for the understanding of Neandertal extinction, Journal of World Prehistory, vol. 13, p. 1-68.

ZILHÃO J., d'ERRICO F. (2003a) - The chronology of the Aurignacian and Transitional technocomplexes. Where do we stand?, in J. Zilhão et F. d'Errico dir., The chronology of the Aurignacian and of the transitional technocomplexes. Dating, stratigraphies, cultural implications, Trabalhos de Arqueologia, 33, Instituto Português de Arqueologia, Lisbonne, p. 313-349.

ZILHÃO J., D'ERRICO F. (2003b) - Au Aurignacian "garden of Eden" in southern Germany? An alternative interpretation of the Geissenklösterle and a critique of the Kulturpumpe model, Paléo, t. 15, p. 69-86.

Nicolas TEYSSANDIER

Université Toulouse-Le Mirail

UMR 5608-TRACES, Maison de la Recherche, 5, allées Antonio-Machado 31058 Toulouse Cedex 9 teyssandier@univ-tlse2.fr

Despina LIOLIOS Université Paris X-Nanterre UMR 7055- Préhistoire et Technologie Maison de l'Archéologie et de l'Ethnologie, 92023 Nanterre Cedex despina.liolios@mae.u-paris10.fr 